\title{
For radiation therapy before surgery in esophageal cancer, dose matters, and with each answer comes more questions
}

\author{
Mark Hennon, Sai Yendamuri \\ Department of Thoracic Surgery, Roswell Park Comprehensive Cancer Center, Buffalo, NY, USA \\ Correspondence to: Mark Hennon, MD. Division of Thoracic Surgery, Roswell Park Comprehensive Cancer Center, Elm and Carlton Streets, Buffalo, \\ NY 14263, USA. Email: mark.hennon@roswellpark.org. \\ Provenance: This is an invited article commissioned by the Academic Editor Shuangiiang Li (Department of Thoracic Surgery and West China \\ Medical Center, West China Hospital, Sichuan University, Chengdu, China). \\ Comment on: Semenkovich TR, Samson PP, Hudson JL, et al. Induction Radiation Therapy for Esophageal Cancer: Does Dose Affect Outcomes? \\ Ann Thorac Surg 2019;107:903-11.
}

Submitted Nov 27, 2019. Accepted for publication Dec 08, 2019.

doi: $10.21037 /$ jtd.2019.12.62

View this article at: http://dx.doi.org/10.21037/jtd.2019.12.62

Recently published results from Semenkovich et al. in the March 2019 edition of the Annals of Thoracic Surgery reporting on the impact of induction radiation dosing on outcomes after esophageal resection in patients undergoing tri-modality therapy offer an answer to an important question regarding the treatment of patients with operable esophageal cancer (1). The question being: does dose matter for induction radiation treatment prior to esophagectomy for esophageal cancer. Based on the results reported, the answer is yes, and the conclusions of the authors should help clarify what is the optimal radiation dose prior to esophagectomy. Results from this nicely powered study of patients from the National Cancer Database (NCDB) demonstrate that too high of an induction radiation dose ( $>50.4$ Gy) creates increased risk for perioperative mortality, without any benefit in tumor response, while too small a dose (<30 Gy), does not reduce perioperative mortality, but is associated with worse tumor response. Moderate doses of 45 and 50.4 Gy seemed to offer a sweet spot, with best results noted with $50.4 \mathrm{~Gy}$ in terms of the rate of complete pathologic response. These results were noted for both squamous cell cancer patients and adenocarcinoma patients. Despite known limitations from retrospective studies involving large multi-institution databases that sometime lack granularity, the strength of such large patient numbers coupled with long term survival data from a database that captures over $70 \%$ of cancer cases in the United States should not be overlooked. These results cement the commonly accepted dose range for neoadjuvant therapy for operable esophageal cancer, which is reflected in the fact that from 2004 through 2014 the percentage of patients who received standard dose radiation increased from $70 \%$ to over $90 \%$.

While the emphasis in previous trials for locally advanced esophageal cancer has focused on answering the question of whether neoadjuvant chemoradiotherapy is superior to surgery alone, conflicting results and acknowledged trial flaws allowed for much debate. The Phase III, Dutch CROSS trial helped put a great deal of that debate to rest, and long-term results from this welldesigned trial offer compelling evidence for the superiority of chemoradiotherapy prior to surgery compared to surgery alone (2). There is now widespread acceptance for neoadjuvant chemoradiotherapy prior to esophagectomy for patients with locally advanced but surgically resectable disease, and this is reflected in NCCN guidelines. The results from Semenkovich et al. go one step further and help clarify the best radiation dose.

An interesting follow up to the question of optimal dosing for induction radiation therapy will be whether radiation is needed or beneficial for all patients undergoing induction therapy. Is it a combination of the systemic therapy and local radiation therapy that improves long term outcomes, or just the systemic therapy? Similar to methods analyzing the effectiveness of chemoradiotherapy followed by surgery versus surgery alone, trials compared 
neoadjuvant chemotherapy followed by surgery to those undergoing surgery alone (3). A significant benefit for the induction chemotherapy arm was noted compared to those undergoing surgery alone. In an era of personalized medicine with an increasing emphasis on understanding tumor biology, a patient's long-term outcome may very well be more dependent on the tumor response to systemic therapy. It is not unreasonable to now question whether chemoradiotherapy prior to surgery is superior to chemotherapy alone followed by surgery.

Chemoradiotherapy is commonly administered with the intent of achieving maximal induction response. Given that chemoradiation has been shown to increase the rate of complete pathologic response, and that long-term survival is best in those achieving a complete pathologic response, logic dictates that long term survival should be improved with the addition of induction radiation therapy; except that no randomized controlled trial has ever demonstrated a survival advantage with neoadjuvant chemoradiation $v$ s. chemotherapy when the two modalities have been directly compared to each other for treatment of adenocarcinoma (4). Further, two recently published analyses of the NCDB showed no benefit in long term survival for patients undergoing chemoradiotherapy compared to chemotherapy alone prior to surgery $(5,6)$.

How could this be? Results of published meta-analyses examining different treatment approaches, despite having strength in total number of patients, are limited by variation in study methodologies, systemic chemotherapy regimens, radiation dosing, and surgical technique. In contrast, large multi-institutional databases can be analyzed for specific periods of time, capturing large patient numbers, thereby providing long term survival results for contemporary treatment regimens. Given the high prevalence of nodal involvement for esophageal cancer, long term results may be more dependent on response to systemic therapy, and not local treatment effect resulting from the addition of radiation. The more we understand about tumor biology, the more we may see treatments that target a tumor at the molecular level impact long term survival.

Cite this article as: Hennon $M$, Yendamuri S. For radiation therapy before surgery in esophageal cancer, dose matters, and with each answer comes more questions. J Thorac Dis 2019;11(12):5662-5663. doi: 10.21037/jtd.2019.12.62

\section{Acknowledgments}

None.

\section{Footnote}

Conflicts of Interest: The authors have no conflicts of interest to declare.

Ethical Statement: The authors are accountable for all aspects of the work in ensuring that questions related to the accuracy or integrity of any part of the work are appropriately investigated and resolved.

\section{References}

1. Semenkovich TR, Samson PP, Hudson JL, et al. Induction Radiation Therapy for Esophageal Cancer: Does Dose Affect Outcomes? Ann Thorac Surg 2019;107:903-11.

2. Shapiro J, van Lanschot JJB, Hulshof MCCM, et al. Neoadjuvant chemoradiotherapy plus surgery versus surgery alone for oesophageal or junctional cancer (CROSS): long-term results of a randomised controlled trial. Lancet Oncol 2015;16:1090-8.

3. Cunningham D, Allum WH, Stenning SP, et al. Preoperative chemotherapy versus surgery alone for resectable gastroesophageal cancer. N Engl J Med 2006:355;11-20.

4. Stahl M, Walz MK, Stuschke M, et al. Phase III comparison of preoperative chemotherapy compared with chemoradiotherapy in patients with locally advanced adenocarcinoma of the esophagogastric junction. J Clin Oncol 2009;27:851-6.

5. Al-Sukhni E, Gabriel E, Attwood K, et al. No survival Difference with Neoadjuvant Chemoradiotherapy in Resectable Esophageal and Gastroesophageal Junction Adenocarcinoma: Results from the National Cancer Database. J Am Coll Surg 2016;223:784-792.e1.

6. Miller JA, Groman A, Yendamuri S, et al. Radiation With Neoadjuvant Chemotherapy Does Not Improve Outcomes in Esophageal Squamous Cell Cancer. J Surg Res 2019:236:259-65. 